\title{
Linear Complexity and Autocorrelation of Prime Cube Sequences
}

\author{
Young-Joon Kim, Seok-Yong Jin, and Hong-Yeop Song \\ Department of Electrical and Electronic Engineering \\ Yonsei University, Seoul, 121-749, Korea \\ \{yj.kim, sy.jin, hysong\}@yonsei.ac.kr
}

\begin{abstract}
We review a binary sequence based on the generalized cyclotomy of order 2 with respect to $p^{3}$, where $p$ is an odd prime. Linear complexities, minimal polynomials and autocorrelation of these sequences are computed.
\end{abstract}

\section{Introduction}

Let $n \geq 2$ be a positive integer and $Z_{n}^{*}$ be the multiplicative group of the integer ring $Z_{n}$. For a partition $\left\{D_{i} \mid i=0,1, \cdots, d-1\right\}$ of $Z_{n}^{*}$, if there exist elements $g_{1}, \cdots, g_{d}$ of $Z_{n}^{*}$ satisfying $D_{i}=g_{i} D_{0}$ for all $i$ where $D_{0}$ is a multiplicative subgroup of $Z_{n}^{*}$, the $D_{i}$ are called generalized cyclotomic classes of order $d$. In 1998, Ding and Helleseth [1] introduced the new generalized cyclotomy with respect to $p_{1}^{e_{1}} \cdots p_{t}^{e_{t}}$ and defined a balanced binary sequence based on their own generalized cyclotomy, where $p_{1}, \cdots, p_{t}$ are distinct odd primes and $e_{1}, \cdots, e_{t}$ are positive integers. Before them, there have been lots of studies about cyclotomy, but they are only about ones with respect to $p$ or $p^{2}$ or $p q$ where $p$ and $q$ are distinct odd primes 147/8. In [1] they also introduced how to construct a balanced binary sequence based on their generalized cyclotomy. Let it call the generalized cyclotomic sequences. Those sequences includes the binary quadratic residue sequences also known as Legendre Sequences because these sequences can be understood as the generalized cyclotomic sequences with respect to $p$.

In 1998, C. Ding 4 presented some cyclotomy sequences with period $p^{2}$ which are not balanced. They are defined in a slightly different way from the generalized cyclotomic sequences with respect to $p^{2}$. In that paper, he calculated the linear complexities with minor errors. Y.-H. Park and others [5] corrected the errors. The linear complexity of the sequence is not so good. In general, the linear complexity of a sequence is considered as good when it is not less than half of the period of the sequence. Recently, in [7, Yan et al. calculated the linear complexity and autocorrelation of generalized cyclotomic sequences of order 2 with respect to $p^{2}$.

In this paper, we compute the linear complexity and autocorrelation of the generalized cyclotomic sequences with respect to $p^{3}$. Hereafter we will call these sequences as prime cube sequences. 


\section{Prime Cube Sequences}

Let $p$ be an odd prime. Let $g$ be a primitive root of $p^{2}$. Then it's well known that $g$ is also a primitive root of $p^{k}$ for $k \geq 1[2]$. The order of $g$ modulo $p$ is $p-1$, the order of $g$ modulo $p^{2}$ is $p(p-1)$ and the order of $g$ modulo $p^{3}$ is $p^{2}(p-1)$.

Define

$$
\begin{aligned}
& D_{0}^{(p)}=\left(g^{2}\right) \quad(\bmod p) \quad D_{1}^{(p)}=g D_{0}^{(p)} \quad(\bmod p) \\
& D_{0}^{\left(p^{2}\right)}=\left(g^{2}\right) \quad\left(\bmod p^{2}\right) D_{1}^{\left(p^{2}\right)}=g D_{0}^{\left(p^{2}\right)} \quad\left(\bmod p^{2}\right) \\
& D_{0}^{\left(p^{3}\right)}=\left(g^{2}\right) \quad\left(\bmod p^{3}\right) D_{1}^{\left(p^{3}\right)}=g D_{0}^{\left(p^{3}\right)} \quad\left(\bmod p^{3}\right)
\end{aligned}
$$

Then $Z_{p}^{*}=D_{0}^{(p)} \cup D_{1}^{(p)}, Z_{p^{2}}^{*}=D_{0}^{\left(p^{2}\right)} \cup D_{1}^{\left(p^{2}\right)}$ and $Z_{p^{3}}^{*}=D_{0}^{\left(p^{3}\right)} \cup D_{1}^{\left(p^{3}\right)}$. For $i=0,1,2$, the $D_{j}^{\left(p^{i}\right)}$ are called generalized cyclotomic classes of order 2 with respect to $p^{j}$. Note that

$$
Z_{p^{3}}=D_{0}^{\left(p^{3}\right)} \cup D_{1}^{\left(p^{3}\right)} \cup p D_{0}^{\left(p^{2}\right)} \cup p D_{1}^{\left(p^{2}\right)} \cup p^{2} D_{0}^{(p)} \cup p^{2} D_{1}^{(p)} \cup\{0\} .
$$

Here and hereafter, $\frac{p^{3}}{p^{i}} D_{j}^{\left(p^{i}\right)}$ are sets of elements obtained by multiplying $\frac{p^{3}}{p^{i}}$ to the elements of $D_{j}^{\left(p^{i}\right)}$ over $Z_{p^{3}}$ for $i=0,1,2$ and $j=0,1$.

In [1], the authors define the binary prime cube sequence $\{s(n)\}$ as follows 1$]$ :

$$
s(i)= \begin{cases}0, & \text { if }\left(i \bmod p^{3}\right) \in C_{0} \\ 1, & \text { if }\left(i \bmod p^{3}\right) \in C_{1} .\end{cases}
$$

where $C_{0}=\bigcup_{d \mid p^{3}, d>1} \frac{p^{3}}{d} D_{0}^{(d)}$ and $C_{1}=\{0\} \cup \bigcup_{d \mid p^{3}, d>1} \frac{p^{3}}{d} D_{1}^{(d)}$.

\section{Linear Complexity and Minimal Polynomial}

Let $\{s(n)\}$ be a sequence of period $L$ over a field $F$. The linear complexity of $\{s(n)\}$ is defined to be least positive integer $l$ such that there are constants $c_{0}=1, c_{1}, \cdots, c_{l} \in F$ satisfying

$$
-s(i)=c_{1} s(i-1)+c_{2} s(i-2)+\cdots+c_{l} s(i-l) \text { for all } l \leq i<L
$$

The polynomial $c(x)=c_{0}+c_{1} x+\cdots+c_{l} x^{l}$ is called a minimal polynomial of $\{s(n)\}$. Let $\{s(n)\}$ be a sequence of period $L$ over a field $F$, and $S(x)=$ $s(0)+s(1) x+\cdots+s(L-1) x^{L-1}$. It is well known that 3

1. the mimimal polynomial of $\{s(n)\}$ is given by

$$
c(x)=\left(x^{L}-1\right) / \operatorname{gcd}\left(x^{L}-1, S(x)\right)
$$

2. the linear complexity of $\{s(n)\}$ is given by

$$
C_{L}=L-\operatorname{deg}\left(\operatorname{gcd}\left(x^{L}-1, S(x)\right)\right)
$$


Lemma 1. For $a \in Z_{p^{3}}^{*}$ and $1 \leq i \leq 3$

$$
a D_{0}^{\left(p^{i}\right)}=\left\{\begin{array}{ll}
D_{0}^{\left(p^{i}\right)}, & \text { if } a \in D_{0}^{\left(p^{i}\right)} \\
D_{1}^{\left(p^{i}\right)}, & \text { if } a \in D_{1}^{\left(p^{i}\right)}
\end{array} \quad, \quad a D_{1}^{\left(p^{i}\right)}=\left\{\begin{array}{ll}
D_{1}^{\left(p^{i}\right)}, & \text { if } a \in D_{0}^{\left(p^{i}\right)} \\
D_{0}^{\left(p^{i}\right)}, & \text { if } a \in D_{1}^{\left(p^{i}\right)}
\end{array}\right. \text {. }\right.
$$

Proof. It can be proved in the same way as [4.

Lemma 2. Let $b$ be any integer. Then $D_{i}^{\left(p^{3}\right)}+b p=D_{i}^{\left(p^{3}\right)}$ and $D_{i}^{\left(p^{2}\right)}+b p=D_{i}^{\left(p^{2}\right)}$ for $i=0,1$.

Proof. It can also be proved in the same way as [4].

Lemma 3. $-1\left(\bmod p^{3}\right) \in D_{0}^{\left(p^{3}\right)}$ if and only if $-1\left(\bmod p^{2}\right) \in D_{0}^{\left(p^{2}\right)}$ if and only if $-1(\bmod p) \in D_{0}^{(p)}$ if and only if $p \equiv 1(\bmod 4)$.

Proof. It is well known that $-1(\bmod p) \in D_{0}^{(p)}$ if and only if $p \equiv 1(\bmod 4)[2$. Using Lemma 2, we can show $-1(\bmod p) \in D_{0}^{(p)}$ implies $-1\left(\bmod p^{2}\right) \in D_{0}^{\left(p^{2}\right)}$ and $-1\left(\bmod p^{3}\right) \in D_{0}^{\left(p^{3}\right)}$. The converse is obvious.

Lemma 4. $2 \in D_{i}^{\left(p^{3}\right)}$ if and only if $2 \in D_{i}^{\left(p^{2}\right)}$ if and only if $2 \in D_{i}^{(p)}$ for $i=0,1$.

Proof. It can be proved in the same way as [4].

Let $m$ be the order of 2 modulo $p^{3}$ and $\theta$ a primitive $p^{3}$ th root of unity in $G F\left(2^{m}\right)$. Define

$$
S(x)=\sum_{i \in C_{1}} x^{i}=1+\left(\sum_{i \in D_{1}^{\left(p^{3}\right)}}+\sum_{i \in p D_{1}^{\left(p^{2}\right)}}+\sum_{i \in p^{2} D_{1}^{(p)}}\right) x^{i} \in G F(2)[x] .
$$

Then $S(x)$ is generating function of the prime cube sequence $\{s(n)\}$ defined before. To compute $S(\theta)$, we use the generalized cyclotomic numbers of order 2 with respect to $p^{i}$ for $i \geq 1$ defined by

$$
(i, j)_{p^{k}}=\left|\left(D_{i}^{\left(p^{k}\right)}+1\right) \cap D_{j}^{\left(p^{k}\right)}\right| \quad i, j=0,1, \text { and } k=0,1,2 .
$$

Lemma 5. [1] If $p \equiv 3(\bmod 4)$, then

$$
(1,0)_{p^{k}}=(0,0)_{p^{k}}=(1,1)_{p^{k}}=\frac{p^{k-1}(p-3)}{4} \text {, and }(0,1)_{p^{k}}=\frac{p^{k-1}(p+1)}{4} .
$$

If $p \equiv 1(\bmod 4)$, then

$$
(0,1)_{p^{k}}=(1,0)_{p^{k}}=(1,1)_{p^{k}}=\frac{p^{k-1}(p-1)}{4} \text {, and }(0,0)_{p^{k}}=\frac{p^{k-1}(p-5)}{4} .
$$


Note that

$$
0=\theta^{p^{3}}-1=\left(\theta^{p^{2}}\right)^{p}-1=\left(\theta^{p^{2}}-1\right)\left(1+\theta^{p^{2}}+\theta^{2 p^{2}}+\cdots+\theta^{(p-1) p^{2}}\right) .
$$

It follows that

$$
1+\theta^{p^{2}}+\theta^{2 p^{2}}+\cdots+\theta^{(p-1) p^{2}}=1+\sum_{i \in p^{2} D_{0}^{(p)}} \theta^{i}+\sum_{i \in p^{2} D_{1}^{(p)}} \theta^{i}=0 .
$$

(3) can be rewritten as follows:

$$
0=\theta^{p^{3}}-1=\left(\theta^{p}\right)^{p^{2}}-1=\left(\theta^{p}-1\right)\left(1+\theta^{p}+\cdots+\theta^{\left(p^{2}-1\right) p}\right) .
$$

It follows that

$$
1+\theta^{p}+\cdots+\theta^{\left(p^{2}-1\right) p}=1+\sum_{i \in p^{2} D_{0}^{(p)} \cup p^{2} D_{1}^{(p)} \cup p D_{0}^{\left(p^{2}\right)} \cup p D_{1}^{\left(p^{2}\right)}} \theta^{i}=0 .
$$

From (4) and (5), we obtain

$$
\sum_{i \in p D_{0}^{\left(p^{2}\right)}} \theta^{i}=\sum_{i \in p D_{1}^{\left(p^{2}\right)}} \theta^{i}
$$

Since $\sum_{i=0}^{p^{3}-1} \theta^{i}=0$, by (5) we obtain

$$
\sum_{i \in D_{0}^{\left(p^{3}\right)}} \theta^{i}=\sum_{i \in D_{1}^{\left(p^{3}\right)}} \theta^{i}
$$

Assume $\theta_{1}=\theta^{p}, \theta_{2}=\theta^{p^{2}}$, then $\theta_{1}$ is a primitive $p^{2}$ th root of unity and $\theta_{2}$ is a primitive $p$ th root of unity in $G F\left(2^{m}\right)$. Define

$$
t_{1}\left(\theta_{1}\right)=\sum_{i \in D_{1}^{\left(p^{2}\right)}} \theta_{1}^{i} \text { and } t_{2}\left(\theta_{2}\right)=\sum_{i \in D_{1}^{(p)}} \theta_{2}^{i} .
$$

Lemma 6. 55 $\sum_{i \in p Z_{p}} \theta_{1}^{i}+\sum_{i \in D_{1}^{\left(p^{2}\right)}} \theta_{1}^{i}=0$ if $p$ is an odd prime.

Lemma 7. $\sum_{i \in D_{0}^{\left(p^{2}\right)}} \theta_{1}^{i}=\sum_{i \in D_{1}^{\left(p^{2}\right)}} \theta_{1}^{i}=t_{1}\left(\theta_{1}\right)=0$.

Proof. From (44), (6) and Lemma 6, obvious.

Lemma 8. [6] $t_{2}\left(\theta_{2}\right) \in\{0,1\}$ if and only if $2 \in D_{0}^{(p)}$ if and only if $p \equiv \pm 1(\bmod 8)$

Lemma 9. Let the symbols be the same as before,

$$
S\left(\theta^{a}\right)= \begin{cases}\frac{p+1}{2} \quad(\bmod 2), & \text { if } a=0 \\ S(\theta), & \text { if } a \in D_{0}^{\left(p^{3}\right)} \\ S(\theta)+1, & \text { if } a \in D_{1}^{\left(p^{3}\right)} \\ \frac{p+1}{2}+t_{2}\left(\theta_{2}\right), & \text { if } a \in p D_{0}^{\left(p^{2}\right)} \\ \frac{p-1}{2}+t_{2}\left(\theta_{2}\right), & \text { if } a \in p D_{1}^{\left(p^{2}\right)} \\ 1+t_{2}\left(\theta_{2}\right), & \text { if } a \in p^{2} D_{0}^{(p)} \\ t_{2}\left(\theta_{2}\right), & \text { if } a \in p^{2} D_{1}^{(p)} .\end{cases}
$$


Proof. For the case $a=0$, we have $S\left(\theta^{a}\right)=S(1)=\frac{p^{3}+1}{2} \equiv \frac{p+1}{2}(\bmod 2)$. If $a \in D_{0}^{\left(p^{3}\right)}$, by definition there is an integer $s$ such that $a=g^{2 s}$. It follows that

$$
\begin{array}{r}
a D_{1}^{\left(p^{3}\right)}=\left\{g^{2 s+2 t+1} \mid t=0,1, \cdots, p^{2}(p-1)-1\right\}=D_{1}^{\left(p^{3}\right)} \\
a p D_{1}^{\left(p^{2}\right)}=p\left\{g^{2 s+2 t+1} \mid t=0,1, \cdots, p(p-1)-1\right\}=p D_{1}^{\left(p^{2}\right)} \\
a p^{2} D_{1}^{(p)}=p^{2}\left\{g^{2 s+2 t+1} \mid t=0,1, \cdots,(p-1)-1\right\}=p^{2} D_{1}^{\left(p^{2}\right)} .
\end{array}
$$

Hence

$$
S\left(\theta^{a}\right)=1+\left(\sum_{i \in D_{1}^{\left(p^{3}\right)}}+\sum_{i \in p D_{1}^{\left(p^{2}\right)}}+\sum_{i \in p^{2} D_{1}^{(p)}}\right) \theta^{a i}=1+\left(\sum_{i \in D_{1}^{\left(p^{3}\right)}}+\sum_{i \in p D_{1}^{\left(p^{2}\right)}}+\sum_{i \in p^{2} D_{1}^{(p)}}\right) \theta^{i}=S(\theta) .
$$

If $a \in D_{1}^{\left(p^{3}\right)}$, then $a D_{1}^{\left(p^{3}\right)}=D_{0}^{\left(p^{3}\right)}, a p D_{1}^{\left(p^{2}\right)}=p D_{0}^{\left(p^{2}\right)}, a p^{2} D_{1}^{(p)}=p^{2} D_{0}^{\left(p^{2}\right)}$. By (44), (6) and (7)

$$
S\left(\theta^{a}\right)=1+\left(\sum_{i \in D_{0}^{\left(p^{3}\right)}}+\sum_{i \in p D_{0}^{\left(p^{2}\right)}}+\sum_{i \in p^{2} D_{0}^{(p)}}\right) \theta^{i}=S(\theta)+1 .
$$

Note that $D_{1}^{\left(p^{3}\right)} \bmod p=D_{1}^{(p)},\left|D_{1}^{\left(p^{3}\right)}\right|=p^{2}\left|D_{1}^{(p)}\right|, \theta_{1}^{p^{2}}=1$ and $\theta_{2}^{p}=1$.

For $a=a_{1} p, a_{1} \in Z_{p^{2}}^{*}=D_{0}^{\left(p^{2}\right)} \cup D_{1}^{\left(p^{2}\right)}$, we have

$$
\begin{aligned}
S\left(\theta^{a}\right) & =1+\left(\sum_{i \in D_{1}^{\left(p^{3}\right)}}+\sum_{i \in p D_{1}^{\left(p^{2}\right)}}+\sum_{i \in p^{2} D_{1}^{(p)}}\right) \theta^{a i} \\
& =1+\sum_{i \in D_{1}^{\left(p^{3}\right)}} \theta^{a_{1} p i}+\sum_{i \in p D_{1}^{\left(p^{2}\right)}} \theta^{a_{1} p i}+\sum_{i \in p^{2} D_{1}^{(p)}} \theta^{a_{1} p i} \\
& =1+\sum_{i \in a_{1} D_{1}^{\left(p^{3}\right)}} \theta_{1}^{i}+\sum_{i \in a_{1} D_{1}^{\left(p^{2}\right)}} \theta_{2}^{i}+\frac{p-1}{2} .
\end{aligned}
$$

If $a_{1} \in D_{0}^{\left(p^{2}\right)}, a_{1} D_{1}^{\left(p^{3}\right)}=D_{1}^{\left(p^{3}\right)}$ and $a_{1} D_{1}^{\left(p^{2}\right)}=D_{1}^{\left(p^{2}\right)}$. we have

$$
\begin{aligned}
S\left(\theta^{a}\right) & =\frac{p+1}{2}+\sum_{i \in D_{1}^{\left(p^{3}\right)}} \theta_{1}^{i}+\sum_{i \in D_{1}^{\left(p^{2}\right)}} \theta_{2}^{i}=\frac{p+1}{2}+p \sum_{i \in D_{1}^{\left(p^{2}\right)}} \theta_{1}^{i}+p \sum_{i \in D_{1}^{(p)}} \theta_{2}^{i} \\
& =\frac{p+1}{2}+t_{1}\left(\theta_{1}\right)+t_{2}\left(\theta_{2}\right)=\frac{p+1}{2}+t_{2}\left(\theta_{2}\right) .
\end{aligned}
$$

If $a_{1} \in D_{1}^{\left(p^{2}\right)}, a_{1} D_{1}^{\left(p^{3}\right)}=D_{0}^{\left(p^{3}\right)}$ and $a_{1} D_{1}^{\left(p^{2}\right)}=D_{0}^{\left(p^{2}\right)}$. we have

$$
\begin{aligned}
S\left(\theta^{a}\right) & =\frac{p+1}{2}+\sum_{i \in D_{0}^{\left(p^{3}\right)}} \theta_{1}^{i}+\sum_{i \in D_{0}^{\left(p^{2}\right)}} \theta_{2}^{i} \\
& =\frac{p+1}{2}+t_{1}\left(\theta_{1}\right)+1+t_{2}\left(\theta_{2}\right)=\frac{p-1}{2}+t_{2}\left(\theta_{2}\right) .
\end{aligned}
$$


For $a=a_{2} p^{2}, a_{2} \in Z_{p}^{*}=D_{0}^{(p)} \cup D_{1}^{(p)}$, we have

$$
\begin{aligned}
S\left(\theta^{a}\right) & =1+\left(\sum_{i \in D_{1}^{\left(p^{3}\right)}}+\sum_{i \in p D_{1}^{\left(p^{2}\right)}}+\sum_{i \in p^{2} D_{1}^{(p)}}\right) \theta^{a i} \\
& =1+\sum_{i \in a_{2} D_{1}^{\left(p^{3}\right)}} \theta^{p^{2} i}+\sum_{i \in D_{1}^{\left(p^{2}\right)}} \theta_{1}^{a_{2} p^{2} i}+\sum_{i \in D_{1}^{(p)}} \theta_{2}^{a_{2} p^{2} i} \\
& =1+\sum_{i \in a_{2} D_{1}^{\left(p^{3}\right)}} \theta_{2}^{i}+\frac{p^{2}-p}{2}+\frac{p-1}{2} .
\end{aligned}
$$

If $a_{2} \in D_{0}^{(p)}, a_{2} D_{1}^{\left(p^{3}\right)}=D_{1}^{\left(p^{3}\right)}$ and $a_{2} D_{1}^{\left(p^{2}\right)}=D_{1}^{\left(p^{2}\right)}$. we have

$$
S\left(\theta^{a}\right)=\frac{p^{2}+1}{2}+\sum_{i \in D_{1}^{\left(p^{3}\right)}} \theta_{2}^{i}=\frac{p^{2}+1}{2}+p^{2} \sum_{i \in D_{1}^{(p)}} \theta_{2}^{i}=1+t_{2}\left(\theta_{2}\right) .
$$

If $a_{2} \in D_{1}^{\left(p^{2}\right)}, a_{2} D_{1}^{\left(p^{3}\right)}=D_{0}^{\left(p^{3}\right)}$ and $a_{2} D_{1}^{\left(p^{2}\right)}=D_{0}^{\left(p^{2}\right)}$. we have

$$
S\left(\theta^{a}\right)=\frac{p^{2}+1}{2}+\sum_{i \in D_{0}^{\left(p^{3}\right)}} \theta_{2}^{i}=\frac{p^{2}+1}{2}+p^{2} \sum_{i \in D_{0}^{(p)}} \theta_{2}^{i}=t_{2}\left(\theta_{2}\right) .
$$

Define $d_{i}^{\left(p^{3}\right)}(x)=\prod_{a \in D_{i}^{\left(p^{3}\right)}}\left(x-\theta^{a}\right), d_{i}^{\left(p^{2}\right)}(x)=\prod_{a \in D_{i}^{\left(p^{2}\right)}}\left(x-\theta_{1}^{a}\right)$ and $d_{i}^{(p)}(x)=$ $\prod_{a \in D_{i}^{(p)}}\left(x-\theta_{2}^{a}\right), i=0,1$. Then

$$
x^{p^{3}}-1=(x-1) d_{0}^{(p)}(x) d_{1}^{(p)}(x) d_{0}^{\left(p^{2}\right)}(x) d_{1}^{\left(p^{2}\right)}(x) d_{0}^{\left(p^{3}\right)}(x) d_{1}^{\left(p^{3}\right)}(x) .
$$

Lemma 10. $d_{i}^{(p)}(x), d_{i}^{\left(p^{2}\right)}(x), d_{i}^{\left(p^{3}\right)}(x) \in G F(2)[x]$ if and only if $p \equiv \pm 1 \bmod 8$.

Proof. Almost the same proof in 4] can be applied . If $p \equiv \pm 1 \bmod 8$, from Lemma 4 and $8,2 \in D_{0}^{(p)} \cap D_{0}^{\left(p^{2}\right)} \cap D_{0}^{\left(p^{3}\right)}$. Then for $i=0,1,2$, we have

$$
\left.\left(d_{i}^{\left(p^{i}\right)}(x)\right)^{2}=\prod_{a \in D_{i}^{\left(p^{i}\right)}} x^{2}-\theta^{2 p^{i} a}\right)=\prod_{a \in 2 D_{i}^{\left(p^{i}\right)}}\left(x^{2}-\theta^{p^{i} a}\right)=\prod_{a \in D_{i}^{\left(p^{i}\right)}}\left(x^{2}-\theta^{p^{i} a}\right)=d_{i}^{\left(p^{i}\right)}\left(x^{2}\right) .
$$

Thus $d_{i}^{\left(p^{i}\right)}(x) \in G F(2)[x], i=0,1,2$. If $p \equiv \pm 3 \bmod 8$, from Lemma 4 and 8 , $2 \in D_{1}^{(p)} \cap D_{1}^{\left(p^{2}\right)} \cap D_{1}^{\left(p^{3}\right)}$. Then for $i=0,1,2$, we have

$$
\left(d_{i}^{\left(p^{i}\right)}(x)\right)^{2}=\prod_{a \in D_{i+1(\bmod 2)}^{\left(p^{i}\right)}}\left(x^{2}-\theta^{p^{i} a}\right)=d_{i+1(\bmod 2)}^{\left(p^{i}\right)}\left(x^{2}\right) \neq d_{i}^{\left(p^{i}\right)}\left(x^{2}\right) .
$$

Hence $d_{i}^{\left(p^{i}\right)}(x) \notin G F(2)[x], i=0,1,2$. 
Theorem 1. Let $p$ be an odd prime and $\{s(n)\}$ be a prime cube sequence of period $p^{3}$. Then the linear complexity $C_{L}$ of $\{s(n)\}$ is as follows:

$$
C_{L}= \begin{cases}\frac{p^{3}+1}{2}, & \text { if } p \equiv 1 \bmod 8 \\ p^{3}-1, & \text { if } p \equiv 3 \bmod 8 \\ p^{3}, & \text { if } p \equiv 5 \bmod 8 \\ \frac{p^{3}-1}{2}, & \text { if } p \equiv 7 \bmod 8\end{cases}
$$

Proof. If $p \equiv 1 \bmod 8$, from Lemmas $8, t_{2}\left(\theta_{2}\right) \in\{0,1\}$. Furthermore, since $2 \in D_{0}^{(p)} \cap D_{0}^{\left(p^{2}\right)} \cap D_{0}^{\left(p^{3}\right)}$ by Lemma 4 and 8 , $S\left(\theta^{2}\right)=S(\theta)$. Hence, $S(\theta) \in\{0,1\}$. Applying Lemma 9, we have

$$
c(x)=\frac{x^{p^{3}}-1}{\operatorname{gcd}\left(x^{p^{3}}-1, S(x)\right)}=\left\{\begin{array}{l}
(x-1) d_{1}^{\left(p^{3}\right)}(x) d_{0}^{\left(p^{2}\right)}(x) d_{0}^{(p)}(x) \text { if }\left(S(\theta), t_{2}\left(\theta_{2}\right)\right)=(0,0) \\
(x-1) d_{1}^{\left(p^{3}\right)}(x) d_{1}^{\left(p^{2}\right)}(x) d_{1}^{(p)}(x) \text { if }\left(S(\theta), t_{2}\left(\theta_{2}\right)\right)=(0,1) \\
(x-1) d_{0}^{\left(p^{3}\right)}(x) d_{0}^{\left(p^{2}\right)}(x) d_{0}^{(p)}(x) \text { if }\left(S(\theta), t_{2}\left(\theta_{2}\right)\right)=(1,0) \\
(x-1) d_{0}^{\left(p^{3}\right)}(x) d_{1}^{\left(p^{2}\right)}(x) d_{1}^{(p)}(x) \text { if }\left(S(\theta), t_{2}\left(\theta_{2}\right)\right)=(1,1)
\end{array}\right.
$$

It follows that $C_{L}=\operatorname{deg}(c(x))=1+\frac{p^{3}-p^{2}}{2}+\frac{p^{2}-p}{2}+\frac{p-1}{2}=\frac{p^{3}+1}{2}$.

For the cases of $p \equiv 3,5$ and $7 \bmod 8$, we can reach easily by similar procedure with the case $p \equiv 1 \bmod 8$.

\section{Autocorrelation}

The periodic autocorrelation of a binary sequence $\{s(n)\}$ of period $N$ is defined by $C_{s}(\tau)=\sum_{n=0}^{L}(-1)^{s(n+\tau)-s(n)}$ where $0 \leq \tau<L$. Define $d_{s}(i, j ; \tau)=\mid C_{i} \cap$ $\left(C_{j}+\tau\right) \mid, \quad 0 \leq \tau<L, i, j=0,1$

Theorem 2. Let $p$ be an odd prime. Then the autocorrelation profile of the binary prime cube sequence of period $p^{3}$ which is defined at (1) is as follows:

1. $p \equiv 1(\bmod 4)$

$$
C_{s}(\tau)= \begin{cases}p^{3}, & \tau=0 \quad\left(\bmod p^{3}\right) \\ p^{3}-p-3, & \tau \in p^{2} D_{0}^{(p)} \\ p^{3}-p+1, & \tau \in p^{2} D_{1}^{(p)} \\ p^{3}-p^{2}-p-2, & \tau \in p D_{0}^{\left(p^{2}\right)} \\ p^{3}-p^{2}-p+2, & \tau \in p D_{1}^{\left(p^{2}\right)} \\ -p^{2}-2, & \tau \in D_{0}^{\left(p^{3}\right)} \\ -p^{2}+2, & \tau \in D_{1}^{\left(p^{3}\right)}\end{cases}
$$

2. $p \equiv 3(\bmod 4)$

$$
C_{s}(\tau)= \begin{cases}p^{3}, & \tau=0 \quad\left(\bmod p^{3}\right) \\ p^{3}-p-1, & \tau \in p^{2} D_{0}^{(p)} \cup p^{2} D_{1}^{(p)} \\ p^{3}-p^{2}-p, & \tau \in p D_{0}^{\left(p^{2}\right)} \cup p D_{1}^{\left(p^{2}\right)} \\ -p^{2}, & \tau \in D_{0}^{\left(p^{3}\right)} \cup D_{1}^{\left(p^{3}\right)}\end{cases}
$$


Proof. Since $C_{s}(\tau)=p^{3}-4 d_{s}(1,0 ; \tau)$, we need to calculate $d_{s}(1,0 ; \tau)$. Note that

$$
\begin{aligned}
d_{s}(1,0 ; \tau) & =\left|C_{1} \cap\left(C_{0}+\tau\right)\right| \\
& =\left|C_{1} \cap\left(p^{2} D_{0}^{(p)}+\tau\right)\right|+\left|C_{1} \cap\left(p D_{0}^{\left(p^{2}\right)}+\tau\right)\right|+\left|C_{1} \cap\left(D_{0}^{\left(p^{3}\right)}+\tau\right)\right|
\end{aligned}
$$

Denote the first, the second and the third term in (8) as $A(\tau), B(\tau)$ and $C(\tau)$, respectively. To begin with, we are going to compute $A(\tau)$. Note that

$$
\begin{aligned}
A(\tau) & =\left|C_{1} \cap\left(p^{2} D_{0}^{(p)}+\tau\right)\right|=\left|\{0\} \cap\left(p^{2} D_{0}^{(p)}+\tau\right)\right|+\left|p^{2} D_{1}^{(p)} \cap\left(p^{2} D_{0}^{(p)}+\tau\right)\right| \\
& +\left|p D_{1}^{\left(p^{2}\right)} \cap\left(p^{2} D_{0}^{(p)}+\tau\right)\right|+\left|D_{1}^{\left(p^{3}\right)} \cap\left(p^{2} D_{0}^{(p)}+\tau\right)\right| .
\end{aligned}
$$

Denote the first, the second, the third and the fourth term in (9) as $A_{1}(\tau)$, $A_{2}(\tau), A_{3}(\tau)$ and $A_{4}(\tau)$, respectively. Let us compute $A_{1}(\tau)$ first. When $\tau=0$, $A_{1}(\tau)=\left|\{0\} \cap p^{2} D_{0}^{(p)}\right|=0$. When $\tau \in p D_{i}^{\left(p^{2}\right.}$ for $i=0,1$, by Lemma 2, any element of $p^{2} D_{0}^{(p)}+\tau$ is an element of $p D_{i}^{\left(p^{2}\right)}$ for $i=0,1$, respectively. Similarly, when $\tau \in D_{i}^{\left(p^{3}\right)}$ for $i=0,1$, any element of $p^{2} D_{0}^{(p)}+\tau$ is an element of $D_{i}^{\left(p^{3}\right)}$ for $i=0,1$, respectively. Therefore, when $\tau \in\{0\} \cup p D_{0}^{\left(p^{2}\right)} \cup p D_{1}^{\left(p^{2}\right)} \cup D_{0}^{\left(p^{3}\right)} \cup D_{1}^{\left(p^{3}\right)}$, $A_{1}(\tau)=0$. Next thing to do is to compute the value of $A_{1}(\tau)$ when $\tau$ belongs to the set $p^{2} D_{0}^{(p)} \cup p^{2} D_{1}^{(p)}$. From Lemma 1 and 3 if $p \equiv 1 \bmod 4, \tau \in p^{2} D_{i}^{(p)}$ implies $-\tau \in p^{2} D_{i}^{(p)}$ for $i=0,1$, respectively. Hence, in this case, $A_{1}(\tau)=1$ if $\tau \in p^{2} D_{0}^{(p)}$ and $A_{1}(\tau)=0$ if $\tau \in p^{2} D_{1}^{(p)}$. Likewise if $p \equiv 1 \bmod 4, \tau \in p^{2} D_{i}^{(p)}$ implies $-\tau \in p^{2} D_{i+1 \bmod 2}^{(p)}$ for $i=0,1$, respectively. Hence, $A_{1}(\tau)=0$ if $\tau \in p^{2} D_{0}^{(p)}$ and $A_{1}(\tau)=1$ if $\tau \in p^{2} D_{1}^{(p)}$. Summarizing these, we have

$$
A_{1}(\tau)=\left\{\begin{array}{l}
0, \tau \in\{0\} \cup p D_{0}^{\left(p^{2}\right)} \cup p D_{1}^{\left(p^{2}\right)} \cup D_{0}^{\left(p^{3}\right)} \cup D_{1}^{\left(p^{3}\right)} \\
1, \tau \in p^{2} D_{0}^{(p)} \text { and } p \equiv 1 \bmod 4 \\
0, \tau \in p^{2} D_{0}^{(p)} \text { and } p \equiv 3 \bmod 4 \\
0, \tau \in p^{2} D_{1}^{(p)} \text { and } p \equiv 1 \bmod 4 \\
1, \tau \in p^{2} D_{1}^{(p)} \text { and } p \equiv 3 \bmod 4
\end{array}\right.
$$

Next let us consider $A_{2}(\tau)$. Similarly $A_{2}(\tau)=0$ if $\tau \in\{0\} \cup p D_{0}^{\left(p^{2}\right)} \cup p D_{1}^{\left(p^{2}\right)} \cup$ $D_{0}^{\left(p^{3}\right)} \cup D_{1}^{\left(p^{3}\right)}$. When $\tau \in p^{2} D_{0}^{(p)} \cup p^{2} D_{1}^{(p)}, A_{2}(\tau)=\left|p^{2} D_{1}^{(p)} \cap\left(p^{2} D_{0}^{(p)}+\tau\right)\right|=$ $\left|p^{2} D_{1}^{(p)} \cap\left(p^{2} D_{0}^{(p)}+p^{2} a\right)\right|$ for some $a \in D_{0}^{(p)} \cup D_{1}^{(p)}$. Therefore $A_{2}(\tau)=\mid D_{1}^{(p)} \cap$ $\left(D_{0}^{(p)}+a\right)|=| a^{-1} D_{1}^{(p)} \cap\left(a^{-1} D_{0}^{(p)}+1\right) \mid$ and by Lemma 1 and the definition of the generalized cyclotomic numbers of order 2 with respect to $p$, we have

$$
A_{2}(\tau)=\left\{\begin{array}{l}
0, \quad \tau \in\{0\} \cup p D_{0}^{\left(p^{2}\right)} \cup p D_{1}^{\left(p^{2}\right)} \cup D_{0}^{\left(p^{3}\right)} \cup D_{1}^{\left(p^{3}\right)} \\
(0,1)_{p}, \tau \in p^{2} D_{0}^{(p)} \\
(1,0)_{p}, \tau \in p^{2} D_{1}^{(p)}
\end{array} .\right.
$$

In the case of $A_{3}(\tau), A_{3}(\tau)=0$ if $\tau \in\{0\} \cup D_{0}^{\left(p^{3}\right)} \cup D_{1}^{\left(p^{3}\right)}$ with the same reason as $A_{1}(\tau)$ and $A_{2}(\tau)$. If $\tau \in \cup p D_{0}^{\left(p^{2}\right)} \cup p D_{1}^{\left(p^{2}\right)}$, then for $i=0,1$, any element 
of $p^{2} D_{i}^{(p)}+\tau$ is a multiple of $p^{2} \bmod p^{3}$ so that it can not be an element of $p D_{1}^{\left(p^{2}\right)}$. Thus, in these cases, $A_{3}(\tau)=0$. In the case of $\tau \in p D_{i}^{\left(p^{2}\right)}$ for $i=0,1$, we have $p^{2} D_{0}^{(p)}+\tau \subset p D_{i}^{\left(p^{2}\right)}$. Therefore, $A_{3}(\tau)=|\emptyset|=0$ if $\tau \in p D_{0}^{\left(p^{2}\right)}$ and $A_{3}(\tau)=\left|p^{2} D_{0}^{(p)}+\tau\right|=\frac{p-1}{2}$ if $\tau \in p D_{1}^{\left(p^{2}\right)}$. Similarly, we can compute $A_{4}(\tau)$. Summarizing these calculation, we have

$$
A_{3}(\tau)=\left\{\begin{array}{ll}
0, & \tau \in Z_{p^{3}} \backslash p D_{1}^{\left(p^{2}\right)} \\
\frac{p-1}{2}, & \tau \in p D_{1}^{\left(p^{2}\right)}
\end{array} \quad A_{4}(\tau)=\left\{\begin{array}{ll}
0, & \tau \in Z_{p^{3}} \backslash D_{1}^{\left(p^{3}\right)} \\
\frac{p-1}{2}, & \tau \in D_{1}^{\left(p^{3}\right)}
\end{array} .\right.\right.
$$

Combining the results of $A_{1}(\tau), A_{2}(\tau), A_{3}(\tau)$ and $A_{4}(\tau)$, we have

$$
A(\tau)=\sum_{1 \leq i \leq 4} A_{i}(\tau)=\left\{\begin{array}{l}
0, \quad \tau=0 \\
\frac{p+3}{4}, \tau \in p^{2} D_{0}^{(p)} \text { and } p \equiv 1 \bmod 4 \\
\frac{p+1}{4}, \tau \in p^{2} D_{0}^{(p)} \text { and } p \equiv 3 \bmod 4 \\
\frac{p-1}{4}, \tau \in p^{2} D_{1}^{(p)} \text { and } p \equiv 1 \bmod 4 \\
\frac{p+1}{4}, \tau \in p^{2} D_{1}^{(p)} \text { and } p \equiv 3 \bmod 4 \\
0, \quad \tau \in p D_{0}^{\left(p^{2}\right)} \\
\frac{p-1}{2}, \tau \in p D_{1}^{\left(p^{2}\right)} \\
0, \quad \tau \in D_{0}^{\left(p^{3}\right)} \\
\frac{p-1}{2}, \tau \in D_{1}^{\left(p^{3}\right)}
\end{array}\right.
$$

Next we are going to compute $B(\tau)$ and $C(\tau)$. Note that

$$
\begin{aligned}
B(\tau) & =\left|\{0\} \cap\left(p D_{0}^{\left(p^{2}\right)}+\tau\right)\right|+\left|p^{2} D_{1}^{(p)} \cap\left(p D_{0}^{\left(p^{2}\right)}+\tau\right)\right| \\
& +\left|p D_{1}^{\left(p^{2}\right)} \cap\left(p D_{0}^{\left(p^{2}\right)}+\tau\right)\right|+\left|D_{1}^{\left(p^{3}\right)} \cap\left(p D_{0}^{\left(p^{2}\right)}+\tau\right)\right| . \\
C(\tau) & =\left|\{0\} \cap\left(D_{0}^{\left(p^{3}\right)}+\tau\right)\right|+\left|p^{2} D_{1}^{(p)} \cap\left(D_{0}^{\left(p^{3}\right)}+\tau\right)\right| \\
& +\left|p D_{1}^{\left(p^{2}\right)} \cap\left(D_{0}^{\left(p^{3}\right)}+\tau\right)\right|+\left|D_{1}^{\left(p^{3}\right)} \cap\left(D_{0}^{\left(p^{3}\right)}+\tau\right)\right| .
\end{aligned}
$$

Denote the first, the second, the third and the fourth term in (12) as $B_{1}(\tau)$, $B_{2}(\tau), B_{3}(\tau)$ and $B_{4}(\tau)$, respectively. Likewise denote the first, the second, the third and the fourth term in (13) as $C_{1}(\tau), C_{2}(\tau), C_{3}(\tau)$ and $C_{4}(\tau)$, respectively. With almost the same way, we can reach the following:

\begin{tabular}{|c|ccccc|}
\hline$p \equiv 1 \bmod 4$ & $B_{1}(\tau)$ & $B_{2}(\tau)$ & $B_{3}(\tau)$ & $B_{4}(\tau)$ & $B(\tau)$ \\
\hline$\tau \in p D_{0}^{\left(p^{2}\right)}$ & 1 & $\frac{p-1}{2}$ & $(0,1)_{p^{2}}$ & 0 & $\frac{p^{2}+p+2}{4}$ \\
\hline$\tau \in p D_{1}^{\left(p^{2}\right)}$ & 0 & 0 & $(1,0)_{p^{2}}$ & 0 & $\frac{p(p-1)}{4}$ \\
\hline$\tau \in D_{1}^{\left(p^{3}\right)}$ & 0 & 0 & 0 & $\frac{p^{2}-p}{2}$ & $\frac{p^{2}-p}{2}$ \\
\hline otherwise & 0 & 0 & 0 & 0 & 0 \\
\hline
\end{tabular}




\begin{tabular}{|c|cccc|c|}
\hline$p \equiv 3 \bmod 4$ & $B_{1}(\tau)$ & $B_{2}(\tau)$ & $B_{3}(\tau)$ & $B_{4}(\tau)$ & $B(\tau)$ \\
\hline$p D_{0}^{\left(p^{2}\right)}$ & 0 & 0 & $(0,1)_{p^{2}}$ & 0 & $\frac{p(p+1)}{4}$ \\
\hline$\tau \in p D_{1}^{\left(p^{2}\right)}$ & 1 & $\frac{p-1}{2}$ & $(1,0)_{p^{2}}$ & 0 & $\frac{p^{2}-p+2}{4}$ \\
\hline$\tau \in D_{1}^{\left(p^{3}\right)}$ & 0 & 0 & 0 & $\frac{p^{2}-p}{2}$ & $\frac{p^{2}-p}{2}$ \\
\hline otherwise & 0 & 0 & 0 & 0 & 0 \\
\hline
\end{tabular}

By doing the same procedure repeatedly, we can reach the following:

\begin{tabular}{|c|ccccc|c|}
\hline$p \equiv 1 \bmod 4$ & $C_{1}(\tau)$ & $C_{2}(\tau)$ & $C_{3}(\tau)$ & $C_{4}(\tau)$ & $C(\tau)$ \\
\hline$\tau \in D_{0}^{\left(p^{3}\right)}$ & 1 & $\frac{p-1}{2}$ & $\frac{p^{2}-p}{2}$ & $(0,1)_{p^{3}}$ & $\frac{p^{3}+p^{2}+2}{4}$ \\
\hline$\tau \in D_{1}^{\left(p^{3}\right)}$ & 0 & 0 & 0 & $(1,0)_{p^{3}}$ & $\frac{p^{3}-p^{2}}{4}$ \\
\hline otherwise & 0 & 0 & 0 & 0 & 0 \\
\hline
\end{tabular}

\begin{tabular}{|c|cccc|c|}
\hline$p \equiv 3 \bmod 4$ & $C_{1}(\tau)$ & $C_{2}(\tau)$ & $C_{3}(\tau)$ & $C_{4}(\tau)$ & $C(\tau)$ \\
\hline$\tau \in D_{0}^{\left(p^{3}\right)}$ & 0 & 0 & 0 & $(0,1)_{p^{3}}$ & $\frac{p^{3}+p^{2}}{4}$ \\
\hline$\tau \in D_{1}^{\left(p^{3}\right)}$ & 1 & $\frac{p-1}{2}$ & $\frac{p^{2}-p}{2}$ & $(1,0)_{p^{3}}$ & $\frac{p^{3}-p^{2}+2}{4}$ \\
\hline otherwise & 0 & 0 & 0 & 0 & 0 \\
\hline
\end{tabular}

Combining (11), (14), and (16), we can compute $d_{s}(1,0 ; \tau)$. Since $C_{s}(\tau)=$ $p^{3}-4 d_{s}(1,0 ; \tau)$, it completes the proof.

\section{References}

1. Ding, C., Helleseth, T.: New Generalized Cyclotomy and Its Application. Finite Fields and Their Applications 4, 140-166 (1998)

2. Burton, D.M.: Elementary Number Theory, 4th edn. McGraw-Hill, New York (1998)

3. Golomb, S.W.: Shift Register Sequences, Revised edn. Aegean Park Press, Laguna Hills (1982)

4. Ding, C.: Linear Complexity of Some Generalized Cyclotomic Sequences. Int. J. Algebra and Computation 8, 431-442 (1998)

5. Park, Y.H., Hong, D., Chun, E.: On the Linear Complexity of Some Generalized Cyclotomic Sequences. Int. J. Algebra and Computation 14, 431-439 (2004)

6. Cusick, T., Ding, C., Renvall, A.: Stream Ciphers and Number Theory. Elservier Science, Amsterdam (1998)

7. Yan, T., Sun, R., Xiao, G.: Autocorrelation and Linear Complexity of the New Generalized Cyclotomic Sequences. IEICE Trans. Fundamentals E90-A, 857-864 (2007)

8. Bai, E., Liu, X., Xiao, G.: Linear Complexity of New Generalized Cyclotomic Sequences of Order Two of Length pq. IEEE Trans. Inform. Theory 51, 1849-1853 (2005) 